\title{
THE LINEAR TRACE HARNACK QUADRATIC ON A STEADY GRADIENT RICCI SOLITON SATISFIES THE HEAT EQUATION
}

\author{
BENNETT CHOW AND PENG LU \\ (Communicated by Michael Wolf)
}

\begin{abstract}
We show that on steady and shrinking gradient Ricci solitons, expressions involving the linear trace Harnack quadratic satisfy the heat equation. We also interpolate between Li-Yau-type calculations of Cao-Hamilton and Perelman.
\end{abstract}

All quantities shall be assumed $C^{\infty}$.

\section{Evolution of the linear trace Harnack ON STEADY AND SHRINKING GRADIENT RICCI SOLITONS}

Suppose $\left(\mathcal{M}^{n}, g_{i j}(t), h_{i j}(t)\right)$ satisfies the linearized Ricci flows $\frac{\partial}{\partial t} g_{i j}=-2 R_{i j}$ and $\frac{\partial}{\partial t} h_{i j}=\left(\Delta_{L} h\right)_{i j}$, where $\Delta_{L}$ denotes the Lichnerowicz Laplacian. Define the matrix Harnack quantities (see Hamilton [3])

$$
M_{p q}=\Delta R_{p q}-\frac{1}{2} \nabla_{p} \nabla_{q} R+2 R_{p i j q} R^{i j}-R_{p k} R_{q}^{k}, \quad P_{i p q}=\nabla_{i} R_{p q}-\nabla_{p} R_{q i} .
$$

Recall that if $X(t)$ is any time-dependent vector field on $\mathcal{M}$, then the corresponding linear trace Harnack quantity $Z(h, X)=\operatorname{div}(\operatorname{div}(h))+\langle\mathrm{Rc}, h\rangle+2\langle\operatorname{div}(h), X\rangle+$ $h(X, X)$ satisfies (see [2])

$$
\begin{aligned}
\left(\frac{\partial}{\partial t}-\Delta\right) Z= & 2 h^{p q}\left(M_{p q}+2 P_{i p q} X^{i}+R_{p i j q} X^{i} X^{j}\right) \\
& -4\left(\nabla_{j} X^{i}-R_{j}^{i}\right) \nabla^{j}\left(\operatorname{div}(h)_{i}+h_{i k} X^{k}\right) \\
& +2\left(\operatorname{div}(h)_{j}+h_{i j} X^{i}\right)\left(\frac{\partial X^{j}}{\partial t}-\Delta X^{j}-R_{k}^{j} X^{k}\right) \\
& +2 h_{i j}\left(\nabla_{p} X^{i}-R_{p}^{i}\right)\left(\nabla^{p} X^{j}-R^{p j}\right) .
\end{aligned}
$$

Since $\frac{\partial}{\partial t} \mathrm{Rc}=\Delta_{L} \mathrm{Rc}$, we may take $h=\mathrm{Rc}$. Then $2 Z(\mathrm{Rc}, X)=\Delta R+2|\mathrm{Rc}|^{2}+$ $2\langle\nabla R, X\rangle+2 \operatorname{Rc}(X, X)$, which is Hamilton's trace Harnack quadratic. Furthermore, if $\mathrm{Rc}+\nabla \nabla f=0$, then $Z(\mathrm{Rc},-\nabla f)=0$ by $\Delta R+2|\mathrm{Rc}|^{2}=\langle\nabla R, \nabla f\rangle$ and $2 R_{i j} \nabla_{j} f=\nabla_{i} R$.

Received by the editors September 13, 2011.

2010 Mathematics Subject Classification. Primary 53C44.

(C) 2013 American Mathematical Society Reverts to public domain 28 years from publication 
Now consider the linear trace Harnack quantity for the linearized Ricci flow on a steady gradient Ricci soliton.

Lemma 1. If $\left(\mathcal{M}^{n}, g(t), f(t), h(t)\right)$ satisfies $\frac{\partial}{\partial t} g=-2 \mathrm{Rc}=2 \nabla \nabla f, \frac{\partial f}{\partial t}=\Delta f$, $\frac{\partial}{\partial t} h=\Delta_{L} h$, then $Z(h,-\nabla f)=\operatorname{div}(\operatorname{div}(h))+\langle\operatorname{Rc}, h\rangle-2 \operatorname{div}(h)(\nabla f)+h(\nabla f, \nabla f)$ satisfies $\frac{\partial Z}{\partial t}=\Delta Z \square$ That is, the linear trace Harnack quantity solves the heat equation.

Proof. By $M_{p q}=P_{i p q} \nabla^{i} f, P_{i p q}=R_{p i j q} \nabla^{j} f, \nabla_{j} \nabla^{i} f+R_{j}^{i}=0$, and $\left(\left(\frac{\partial}{\partial t}-\Delta\right) \nabla f\right)^{j}=$ $R_{k}^{j} \nabla^{k} f$, for $Z(h,-\nabla f)$ each of the terms on the right hand side of (1) (with $X=$ $-\nabla f)$ are zero.

Remark. (1) The quantity $Z(h,-\nabla f)$ arises naturally from the following consideration. If $g_{i j}(s), f(s)$ are such that $\frac{\partial}{\partial s} g_{i j}=h_{i j}$ and $\frac{\partial}{\partial s} f=\frac{H}{2}$, where $H=g^{i j} h_{i j}$ (so that $\frac{\partial}{\partial s}\left(e^{-f} d \mu\right)=0$; see [6]), then Perelman's scalar curvature satisfies

$$
\frac{\partial}{\partial s}\left(R+2 \Delta f-|\nabla f|^{2}\right)=Z(h,-\nabla f)-2\langle h, \mathrm{Rc}+\nabla \nabla f\rangle .
$$

Note for a steady gradient Ricci soliton, $\frac{\partial}{\partial s}\left(R+2 \Delta f-|\nabla f|^{2}\right)=Z(h,-\nabla f)$, while $\frac{\partial}{\partial t}\left(e^{-f} d \mu\right)=(-\Delta f-R) e^{-f} d \mu=0$.

(2) If $g(t), f(t)$ solve $\frac{\partial g}{\partial t}=-2 \mathrm{Rc}$ and $\frac{\partial f}{\partial t}=-\Delta f+|\nabla f|^{2}-R$, then $V \doteqdot(2 \Delta f-$ $\left.|\nabla f|^{2}+R\right) e^{-f}$ satisfies $\square^{*} V=-2\left|R_{i j}+\nabla_{i} \nabla_{j} f\right|^{2} e^{-f}$, where $\square^{*}=-\frac{\partial}{\partial t}-\Delta+R$ (see [6]).

The shrinker analogue of Lemma 1 is the following.

Lemma 2. If $\left(\mathcal{M}^{n}, g(t), f(t)\right), t<0$, satisfies $\frac{\partial}{\partial t} g=-2 \mathrm{Rc}=2 \nabla \nabla f+\frac{1}{t} g$, $\frac{\partial f}{\partial t}=|\nabla f|^{2}=\Delta f-\frac{1}{t} f$, and $\frac{\partial}{\partial t} h=\Delta_{L} h$, then $\left(\frac{\partial}{\partial t}-\Delta\right)\left(t^{2}\left(Z(h,-\nabla f)+\frac{H}{2 t}\right)\right)=0$.

Proof. Applying $M_{p q}+\frac{1}{2 t} R_{p q}=P_{i p q} \nabla^{i} f, P_{i p q}=R_{p i j q} \nabla^{j} f, R_{i j}+\nabla_{i} \nabla_{j} f=-\frac{1}{2 t} g_{i j}$, and $\left(\left(\frac{\partial}{\partial t}-\Delta\right) \nabla f\right)^{j}-R_{k}^{j} \nabla^{k} f=-\frac{1}{t} \nabla^{j} f$ to (11) yields $\left(\frac{\partial}{\partial t}-\Delta\right) Z(h,-\nabla f)=$ $-\frac{2}{t} Z(h,-\nabla f)-\frac{1}{t}\langle h, \mathrm{Rc}\rangle-\frac{H}{2 t^{2}}$. Because $\left(\frac{\partial}{\partial t}-\Delta\right) H=2\langle h, \mathrm{Rc}\rangle$, we conclude that

$$
\left(\frac{\partial}{\partial t}-\Delta\right)\left(Z(h,-\nabla f)+\frac{H}{2 t}\right)=-\frac{2}{t}\left(Z(h,-\nabla f)+\frac{H}{2 t}\right) .
$$

Remark. If $h=\mathrm{Rc}$, then for a shrinker, since $\frac{1}{2} \Delta R+|\mathrm{Rc}|^{2}=\frac{1}{2}\langle\nabla R, \nabla f\rangle-\frac{R}{2 t}$ and $H=R$, we have that $Z(h,-\nabla f)+\frac{H}{2 t}=Z(\mathrm{Rc},-\nabla f)+\frac{R}{2 t}=0$.

\section{Interpolating Between Perelman's and CaO-Hamilton's Harnacks ON A STEADY SOLITON}

Now consider the system $\frac{\partial}{\partial t} g_{i j}=-2 R_{i j}=2 \nabla_{i} \nabla_{j} f, \frac{\partial f}{\partial t}=|\nabla f|^{2}$, and $\frac{\partial u}{\partial t}=$ $\Delta u+R u, u>0$ (when $n=2$, this is the linearized Ricci flow) 2 Mimicking Li, Yau, and Hamilton, define $v=\log u, Q=\Delta v+R$, and $L=\frac{1}{2}\left(\frac{\partial}{\partial t}-\Delta\right)-\nabla v \cdot \nabla$. Then

$$
L Q=|\nabla \nabla v|^{2}+\langle\operatorname{Rc}, \nabla \nabla v\rangle+\operatorname{Rc}(\nabla(v-f), \nabla(v-f)) .
$$

\footnotetext{
${ }^{1}$ The same is true if we replace $\frac{\partial f}{\partial t}=\Delta f$ by $\frac{\partial f}{\partial t}=|\nabla f|^{2}$ essentially since $R=-\Delta f=1-|\nabla f|^{2}$.

${ }^{2}$ Note that $\frac{\partial}{\partial t} e^{f}=\Delta e^{f}+R e^{f}$. The trivial case of the following calculations is $v=f$.
} 
Following Cao and Hamilton [1, define the Harnack $P=2 Q+|\nabla v|^{2}+R$. Since

$$
L\left(|\nabla v|^{2}+R\right)=|\mathrm{Rc}|^{2}-|\nabla \nabla v|^{2},
$$

we have that $P=2 \Delta v+|\nabla v|^{2}+3 R$ satisfies the parabolic Bochner-type formula 3

$$
L P=|\nabla \nabla v+\mathrm{Rc}|^{2}+2 \operatorname{Rc}(\nabla(v-f), \nabla(v-f)) .
$$

In particular, if $\mathrm{Rc} \geq 0$, then $L P \geq \frac{1}{n} Q^{2} \geq 0$.

More generally, if $\frac{\partial u}{\partial t}=\varepsilon^{-1} \Delta u+R u$ (interpolating), where $\varepsilon \in \mathbb{R}-\{0\}$, then $P_{\varepsilon}=2 \Delta v+|\nabla v|^{2}+(2 \varepsilon+1) R$ satisfies the heat equation

$$
\begin{aligned}
L_{\varepsilon} P_{\varepsilon}= & \varepsilon^{-1}|\nabla \nabla v|^{2}+2\langle\operatorname{Rc}, \nabla \nabla v\rangle+\varepsilon^{-1}|\operatorname{Rc}|^{2} \\
& +2 \varepsilon^{-1} \operatorname{Rc}(\nabla(v-\varepsilon f), \nabla(v-\varepsilon f))+\left(1-\varepsilon^{-1}\right) \operatorname{Rc}(\nabla(v+f), \nabla(v+f)),
\end{aligned}
$$

where $L_{\varepsilon}=\frac{1}{2}\left(\frac{\partial}{\partial t}-\varepsilon^{-1} \Delta\right)-\varepsilon^{-1} \nabla v \cdot \nabla$. The Ricci terms may also be rewritten as $\left(1+\varepsilon^{-1}\right) \operatorname{Rc}(\nabla(v-f), \nabla(v-f))+2\left(\varepsilon-\varepsilon^{-1}\right) \operatorname{Rc}(\nabla f, \nabla f)$. If $\varepsilon=-1$, we have

$$
\left(\frac{1}{2}\left(\frac{\partial}{\partial t}+\Delta\right)+\nabla v \cdot \nabla\right)\left(2 \Delta v+|\nabla v|^{2}-R\right)=-|\operatorname{Rc}-\nabla \nabla v|^{2}=-|\nabla \nabla(f+v)|^{2} .
$$

This is a special case of Perelman's pointwise energy monotonicity formula. We interpolated the calculations but not the estimates between $\varepsilon^{-1}=-1$ and $\varepsilon^{-1}=1$; for successful interpolations between $\mathrm{Li}-\mathrm{Yau}-\mathrm{Hamilton}$ inequalities, see $\mathrm{Ni}$ [4, [5].

\section{REFERENCES}

[1] Xiaodong Cao and Richard S. Hamilton, Differential Harnack estimates for time-dependent heat equations with potentials, Geom. Funct. Anal. 19 (2009), no. 4, 989-1000, DOI 10.1007/s00039-009-0024-4. MR2570311 (2010j:53124)

[2] Bennett Chow and Richard S. Hamilton, Constrained and linear Harnack inequalities for parabolic equations, Invent. Math. 129 (1997), no. 2, 213-238, DOI 10.1007/s002220050162. MR 1465325(98i:53051)

[3] Richard S. Hamilton, The Harnack estimate for the Ricci flow, J. Differential Geom. 37 (1993), no. 1, 225-243. MR 1198607 (93k:58052)

[4] Lei Ni, A monotonicity formula on complete Kähler manifolds with nonnegative bisectional curvature, J. Amer. Math. Soc. 17 (2004), no. 4, 909-946 (electronic), DOI 10.1090/S08940347-04-00465-5. MR2083471 (2006e:32027)

[5] Lei Ni, A matrix Li-Yau-Hamilton estimate for Kähler-Ricci flow, J. Differential Geom. 75 (2007), no. 2, 303-358. MR2286824 (2008d:53093)

[6] Grisha Perelman, The entropy formula for the Ricci flow and its geometric applications. arXiv:math.DG/0211159.

Department of Mathematics, University of California San Diego, La Jolla, CaliforNIA 92093

Department of Mathematics, University of Oregon, Eugene, Oregon 97403

\footnotetext{
${ }^{3}$ Note that $\nabla \nabla v+\mathrm{Rc}=\nabla \nabla(v-f)$.
} 